- A video clip demonstrating systematic transperineal prostate biopsy is available at www. hkmj.org

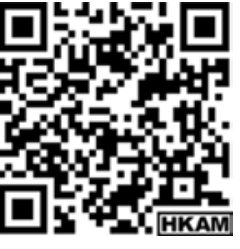

\title{
Picture-in-picture video demonstration of systematic transperineal prostate biopsy
}

\author{
KL Lo *, David Leung, Zoe Lai, Crystal Li, SF Ma, Julius Wong, KK Yuen, Joseph Li, Peter Chiu, \\ SK Mak, Joseph Wong, CF Ng
}

Hong Kong Med J 2021;27:304-5

https://doi.org/10.12809/hkmj208864

We first performed 10-core transperineal prostate biopsy at our institution in 2018. After having acquired the technique and experience, we developed a biopsy protocol that could be modified according to prostate size (Fig): 10 cores for prostate size $<30 \mathrm{cc}, 16$ cores for prostate size 30 to $50 \mathrm{cc}$, and 20 cores for prostate size $>50 \mathrm{cc}$.

We currently take a 24-core prostate biopsy (Fig d), irrespective of prostate size, to further improve the detection of prostate cancer. There is no gold standard protocol for total number of cores required in any patient; it is dictated by hospital policy, availability of resources, and the experience of the urologist.

This video demonstrates systematic transperineal prostate biopsy.

The instruments used during the procedure comprised an ultrasound machine with long sidefire sensor probe, a bed with two leg supports to facilitate lithotomy position, one disposable biopsy gun, two needles for local anaesthesia, two metal trocars, and eight specimen bottles.

Before the procedure and in the absence of any contraindication, patients are prescribed a single dose per oral $1 \mathrm{~g}$ Augmentin and $500 \mathrm{mg}$ ciprofloxacin. One tube of per rectal $4.5 \mathrm{oz}$ Fleet Enema is administered as bowel preparation. Local

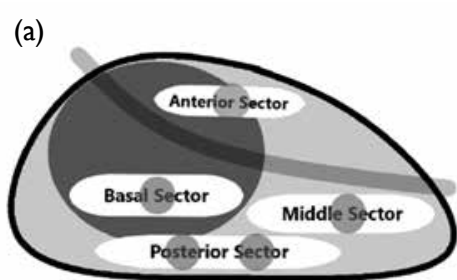

(c)

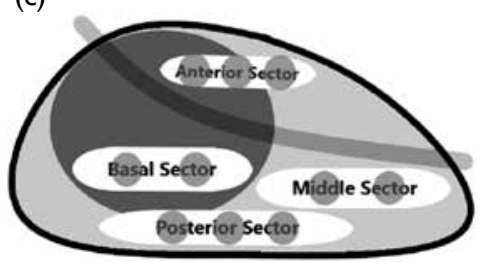

(b)

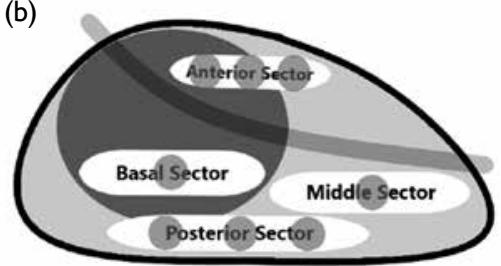

(d)

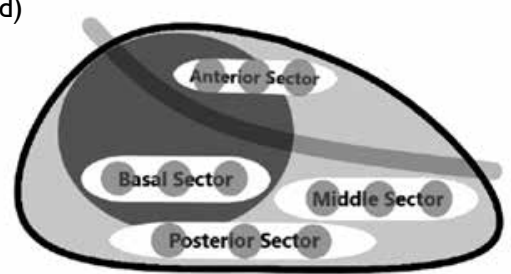

FIG. Templates for prostate biopsy locations, longitudinal view: (a) 10-core template for prostate size $<30 \mathrm{cc}$ (5 cores in each lobe); (b) 16-core template for prostate size $30-50 \mathrm{cc}$ (8 cores in each lobe); (c) 20-core template for prostate size $>50$ cc ( 10 cores in each lobe); (d) 24-core template ( 12 cores in each lobe)

anaesthetic (EMLA) cream is applied to the patient's perineum 1 hour before the procedure.

Step 1: the perineum is disinfected with chlorhexidine.

Step 2: $10 \mathrm{~mL}$ of $1 \%$ lidocaine is injected through each side of the perineum into the periprostatic plane as local anaesthesia, at an angle of $45^{\circ}$ and $15 \mathrm{~mm}$ away from the anus as shown.

It is vital to maintain the needle parallel to the ultrasound probe to ensure continued visualisation of the needle.

Step 3: a 14-gauge metal trocar is inserted through the right side of the perineum under ultrasound guidance.

Step 4: anterior sector prostate biopsies are taken by tilting an 18-gauge disposable biopsy gun downwards.

It is important not to hit the urethra during the procedure.

Step 5: basal sector prostate biopsies are taken. taken.

Step 6: central sector prostate biopsies are

Step 7: posterior sector prostate biopsies are taken.

Step 8: Left lobe prostate biopsies are also taken using a technique similar to that for the right lobe.

Final step: After removing the metal trocars, haemostasis is achieved by compression. A transparent adhesive film dressing is sprayed over the two puncture sites.

No post-procedure antibiotic is required.

\section{Author contributions}

All authors contributed to the concept or design of the study, acquisition of the data, analysis or interpretation of the data, drafting of the manuscript, and critical revision of the manuscript for important intellectual content. All authors had full access to the data, contributed to the study, approved 
the final version for publication, and take responsibility for its written informed consent. accuracy and integrity.

\section{Conflicts of interest}

KL Lo *, FHKAM (Surgery)

The authors have no conflicts of interest to disclose.

D Leung, MRCS

Z Lai, RN

C Li, RN

SF Ma, MB, ChB

J Wong, MRCS

KK Yuen, FHKAM (Surgery)

\section{Funding/support}

J Li, FHKAM (Surgery)

This study received no specific grant from any funding agency in the public, commercial, or not-for-profit sectors.

P Chiu, FHKAM (Surgery)

SK Mak, FHKAM (Surgery)

J Wong, FHKAM (Surgery)

\section{Ethics approval}

CF Ng, FHKAM (Surgery)

This study was approved by the Joint Chinese University of Hong Kong-New Territories East Cluster Clinical Research Ethics Committee (Ref CREC 2018.323). The patient provided

Department of Surgery, The Chinese University of Hong Kong, Hong Kong

* Corresponding author: lokalun@surgery.cuhk.edu.hk 